\title{
VO-KHAC KHOAN PHONG TUAN NGHIEM Étude sur les aspects théorique et pratique de la segmentation aux moindres carrés
}

Revue française d'automatique, d'informatique et de recherche opérationnelle. Recherche opérationnelle, tome 2, no V1 (1968), p. 77-90.

<http://www.numdam.org/item?id=RO_1968_2_1_77_0>

() AFCET, 1968, tous droits réservés.

L'accès aux archives de la revue « Revue française d'automatique, d'informatique et de recherche opérationnelle. Recherche opérationnelle » implique l'accord avec les conditions générales d'utilisation (http://www.numdam.org/ legal.php). Toute utilisation commerciale ou impression systématique est constitutive d'une infraction pénale. Toute copie ou impression de ce fichier doit contenir la présente mention de copyright.

\section{Numdam}

Article numérisé dans le cadre du programme

Numérisation de documents anciens mathématiques

http://www.numdam.org/ 
R.I.R.O.

$\left(2^{\mathrm{e}}\right.$ année, № $\mathrm{N}^{\mathrm{O}}, 1968$, p. 77-90)

\title{
ETUDE SUR LES ASPEGTS THEORIQUE ET PRATIQUE DE LA SEGMENTATION AUX MOINDRES GARRES
}

\author{
par Vo Krac Khoan (1) et Nghiem Phong Tuan (2)
}

Résumé. - Pour décrire correctement une population composée d'individus dont certaines caractéristiques sont connues, on peut être amené à la diviser en un petit nombre de groupes homogènes vis-ä-vis d'un ou de plusieurs critères. La division ainsi faite est appelée une segmentation.

Le problème de segmentation se pose, en général, lorsque la population soumise à l'examen est trop nombreuse pour qu'on puisse étudier chacun des individus séparément, et que d'autre part, les caractéristiques auxquelles l'on s'intéresse présentent, dans l'ensemble de la population, une disparité trop grande pour que leur valeur moyenne puisse donner une information utile.

Avec les critères qui permettent de définir l'homogénéité des segments s'introduit nécessairement une notion de ressemblance entre les individus.

On peut considérer alors que le problème de segmentation n'est rien d'autre que celui d'une classification dans laquelle on rassemble les individus qui présentent une grande ressemblance mutuelle.

Les recherches effectuées ont tout d'abord permis de faire la synthèse d'un grand nombre de résultats épars, et de les généraliser; l'étude du problème dans un espace pré-hilbertien permet notamment de prendre en compte les distances généralisées de Mahalanobis et de traiter ainsi correctement le cas de caractères notablement corrélés, ou encore d'appliquer la segmentation à des ensembles de courbes. En précisant le lien qui existe entre cette méthode et l'analyse de variance, elles conduisent à un critère permettant de fixer le nombre optimal de segments. Enfin, des méthodes de calculs ont été mises au point qui apportent dans les cas simples, une solution rigoureuse au problème.

Cet article résume succinctement les aspects théorique et pratique du problème et donne quelques exemples d'application.

\section{INTRODUCTION}

Pour décrire correctement une population composée d'individus dont certaines caractéristiques sont connues, on peut être amené à la diviser en un petit nombre de groupes homogènes vis-à-vis d'un ou de

(1) Professeur associé à la Faculté des Sciences d'Orléans-Tours. Conseiller Scientifique à la Direction des Études, Cegos.

(2) Direction des Études, Cegos. 
plusieurs critères. Les groupes sont appelés ici des segments, conformément au langage utilisé dans les études de marché qui ont donné naissance aux recherches dont les résultats sont exposés dans cet article. La division ainsi faite est appelée une segmentation.

Le problème de segmentation se pose, en général, lorsque la population soumise à l'examen est trop nombreuse pour qu'on puisse étudier chacun des individus séparément, et que d'autre part, les caractéristiques auxquelles l'on s'intéresse présentent, dans l'ensemble de la population, une disparité trop grande pour que leur valeur moyenne puisse donner une information utile.

Avec les critères qui permettent de définir l'homogénéité des segments s'introduit nécessairement une notion de ressemblance entre les individus.

On peut considérer alors que le problème de segmentation n'est rien d'autre que celui d'une classification dans laquelle on rassemble les individus qui présentent une grande ressemblance mutuelle.

La vraie paternité de la segmentation aux moindres carrés doit être attribuée à Dalenius [4]. De nombreuses études ont été entreprises depuis (cf. en particulier, Dalenius et Hodges [5], Cox [3], Fisher [6], MacNaugthon-Smith, Williams et Dale [11], Guinet [8]).

Les recherches effectuées à la direction des Études de la CEGOS (cf. Vo Khac [16] [17] [18], Nghiem [12]) ont tout d'abord permis de faire la synthèse d'un grand nombre de résultats épars, et de les généraliser ; l'étude du problème dans un espace pré-hilbertien permet notamment de prendre en compte les distances généralisées de Mahalanobis et de traiter ainsi correctement le cas de caractères notablement corrélés, ou encore d'appliquer la segmentation à des ensembles de courbes. En précisant le lien qui existe entre cette méthode et l'analyse de variance, elles nous ont conduit au critère permettant de fixer le nombre optimal de segments. Enfin, des méthodes de calculs ont été mises au point qui apportent dans les cas simples, une solution rigoureuse au problème.

Les applications de la segmentation aux moindres carrés sont nombreuses, car ce problème se pose dans presque tous les domaines d'étudè : biométrie [13], psychométrie [15], économétrie [7], organisation [9], publicité [18], classification des pétroles [10], phytosociologie [18], etc.

Cet article résume succinctement les aspects théorique et pratique du problème et donne quelques exemples d'application.

\section{1. - ASPECT THEORIQUE}

\section{1. - Données du problème}

1.1.1. - Espace économique. - Soit $E$ un espace pré-hilbertien [17], c'est-à-dire un espace vectoriel muni d'un produit scalaire, noté \langle|$>$; la norme pré-hilbertienne associée est par définition: $\|x\|=\langle\vec{x}| \vec{x}>1 / 2$. Cet espace pré-hilbertien sera appelé espace économique. 
Dans la plupart des applications pratiques, $E$ est un espace vectoriel à $R$ dimensions. Le produit scalaire est donné par :

$$
<\vec{x} \mid \vec{y}>=\sum_{r, s=1}^{R} \pi_{r s} x^{r} y^{s}
$$

où $x^{r}$ et $y^{z}$ sont les composantes des vecteurs $x$ et $y$, et où $M=\pi_{r s}$ est une matrice carrée symétrique positive, appelée matrice de métrisation.

Pour rendre les différents composants comparables et pour éliminer l'influence de leurs corrélations mutuelles, cette matrice est souvent prise égale à l'inverse de la matrice de leurs variances et co-variances.

1.1.2. - Population. - Soit $\Omega$ un ensemble comportant $N$ éléments $1,2, \ldots, N$. Cet ensemble $\Omega$ s'appelle la population et chaque élément $\omega(\omega=1,2, \ldots, N)$ s'appelle une classe économique.

1.1.3. - Fonction objective et loi de répartition. - A chaque classe économique $\omega$, on fait correspondre :

(i) un vecteur $\vec{y}(\omega) \in E$, appelé vecteur économique (ou objectif);

(ii) un nombre $p(\omega)>0$, appelé mesure de la classe $\omega$ (ou sa probabiblité, ou sa fréquence de répartition).

L'ensemble des vecteurs économiques constitue la fonction économique (ou fonction objective); l'ensemble des nombres $p(\omega)$ constitue la loi de répartition de la population.

\section{2. - Définition des dispersions}

1.2.1. - Dispersion interne. - Soit $\Omega j$ un sous-ensemble de la population $\Omega$. On appelle mesure de $\Omega j$ le nombre $p(\Omega j)=\Sigma p(\omega)$. Le vecteur moyen de la fonction objective sur $\omega \varepsilon \Omega j$ est par définition

$$
\vec{g}(\Omega j)=\frac{1}{p(\Omega j)} \sum_{\omega \in \Omega j} p(\omega) \vec{y}(\omega)
$$

La dispersion interne de $\Omega j$ est alors définie par :

$$
\Delta(\Omega j)=\sum_{\omega \in \Omega j} p(\omega)\|\vec{y}(\omega)-\vec{g}(\Omega j)\|^{2}
$$

1.2.2. - Dispersion de mélange. - On appelle dispersion de mélange des sous-ensembles $\Omega 1, \ldots, \Omega J$ le nombre

$$
\Delta(\Omega 1, \ldots, \Omega J)=\sum_{j=1}^{J} p(\Omega j)\|\vec{g}(\Omega j)-\vec{G}\|^{2}
$$

où $G=g(\Omega)$ désigne le vecteur moyen sur la population. 


\section{3. - Position du problème}

Soit $J$ un nombre fixé, inférieur à $N$. On désire faire une partition de l'ensemble $\Omega$ en $J$ sous-ensembles disjoints non vides $\Omega 1, \ldots, \Omega J$ satisfaisant simultanément aux deux conditions suivantes :

(i) la somme $\Delta(\Omega 1)+\ldots+\Delta(\Omega J)$ des dispersions internes est minimale ;

(ii) la dispersion de mélange $\Delta(\Omega 1, \ldots, \Omega J)$ est maximale.

Une telle partition s'appelle une $J$-segmentation optimale par la méthode des moindres carrés, chaque sous-ensemble $\Omega j(j=1, \ldots, J)$ s'appelle un segment.

La condition (i) exprime l'homogénéité à l'intérieur de chaque segment ; la condition (ii) exprime la différenciation des divers segments.

Notons que la dispersion de mélange joue le même rôle que la quantité d'information [17] dans la théorie informatique, et le même rôle que le pouvoir discriminant dans la méthode de segmentation de Belson-Agostini [1].

\section{4. - Equation de la dispersion}

On peut montrer (cf. par exemple [17]) que :

$$
\Delta(\Omega 1)+\ldots+\Delta(\Omega J)+\Delta(\Omega 1, \ldots, \Omega J)=\Delta(\Omega)
$$

On en déduit que les conditions (i) et (ii) sont compatibles et équivalentes. Le problème posé possède donc au moins une solution.

\section{5. - Critère d'optimalité}

\subsubsection{Distance symbolique entre deux segments}

On appelle distance symbolique entre deux segments $\Omega 1$ et $\Omega 2$ le double de leur dispersion de mélange.

On démontre la relation (cf. [17]) :

$$
d^{2}(\Omega 1, \Omega 2)=2 \Delta(\Omega 1, \Omega 2)=\frac{2 p(\Omega 1) p(\Omega 2)}{p(\Omega 1)+p(\Omega 2)}\|\vec{g}(\Omega 1)-\vec{g}(\Omega 2)\|^{2}
$$

Pour $p(\Omega 1)=p(\Omega 2)=1$, on retrouve la distance ordinaire. Ce n'est pas une distance au sens mathématique du terme parce que les axiomes de la distance ne sont pas vérifiés. Cependant cette notion permet d'exprimer très simplement les critères d'optimalité.

\subsection{2. - Critère de transfert}

Le problème est le suivant :

Soit deux segments $\Omega 1$ et $\Omega 2$ et un groupe de points $S$ qu'il faut mettre en bloc ou bien dans $\Omega 1$, ou bien dans $\Omega 2$. Comment choisir entre $\Omega 1$ 
et $\Omega 2$ pour que la somme des dispersions internes des segments résultants soit la plus petite?

En convenant de mesurer l'éloignement de deux segments par leur distance symbolique, le critère de choix est (cf. [17]) :

On mettra $S$ dans le segment le plus proche.

\subsection{3. - Critère d'appartenance}

Du critère de transfert, on déduit le résultat (cf. [17]) :

Soit $\Omega 1$ et $\Omega 2$ deux segments et $S$ un vrai sous-ensemble de $\Omega 1$. Pour que $\Omega 1$ et $\Omega 2$ appartiennent à une segmentation optimale il est nécessaire que soit vérifiée la relation :

$$
d^{2}(S, \Omega 1-S) \leqslant d^{2}(S, \Omega 2)
$$

\subsection{4. - Propriétés de séparabilité convexe}

La segmentation par la méthode des moindres carrés possède, en particulier la propriété suivante, qu'on peut démontrer à partir du critère d'appartenance (cf. [20]) :

Lorsque la segmentation est optimale, les enveloppes convexes des segments sont deux à deux disjointes.

Il existe d'autres méthodes de segmentations qui ne possèdent pas cette propriété.

\section{6. - Algorithmes de résolution}

Plusieurs algorithmes de résolution ont été étudiés (cf. [20] ou [17]) :

- algorithme de programmation dynamique, pour les problèmes à un seul caractère ;

- algorithme de division hiérarchisée ;

- algorithme d'agglomération hiérarchisée.

Seul ce dernier, qui est le plus simple, dans son principe, sera exposé ici.

\subsection{1. - Première étape}

(i) Phase de calcul. - On établit la matrice initiale (carrée, symétrique, à $N$ colonnes) des distances entre les classes ; pour cela on utilise la formule (6) qui appliquée à deux classes $\omega$ et $\omega$ ! donne :

$$
d^{2}\left(\omega, \omega^{\prime}\right)=\frac{2 p(\omega) p\left(\omega^{\prime}\right)}{p(\omega)+p\left(\omega^{\prime}\right)}\left\|y(\omega)-y\left(\omega^{\prime}\right)\right\|^{2}
$$

(ii) Phase de sélection. - On agglomère les deux classes économiques ayant la plus petite distance. On obtient ainsi une $(N-1)$ segmentation optimale.

1.6.2. - Etape générale. - A partir d'une $I$-segmentation, on veut obtenir une $(I-1)$ segmentation. 
(i) Phase de calcul. - On établit la nouvelle matrice (symétrique, carrée, à I colonne) des distances en utilisant la formule de récurrence suivante (cf. [17]) :

$$
\begin{aligned}
& \mathrm{d}^{2}(X, A+B) \\
& =\frac{[p(X)+p(A)] \mathrm{d}^{2}(X, A)+[p(X)+p(B)] \mathrm{d}^{2}(X, B)-p(X) \mathrm{d}^{2}(A, B)}{p(X)+p(A)+p(B)}
\end{aligned}
$$

(ii) Phase de sélection. - On réunit les deux segments dont la distance est la plus petite.

\section{7. - Choix du nombre de segments}

\subsection{1. - Espace à une dimension}

Lorsque l'espace économique a une seule dimension, l'équation de la dispersion (5) devient celle, bien connue, d'analyse de variance.

Pour un nombre de segments $J$ donné, appelons $\Delta J$ la somme des dispersions internes obtenue.

En analyse de variance, on teste la différenciation entre ce que nous appelons ici des segments, par la méthode de Fisher-Snedecor. Une démarche possible est la suivante :

Soit $N$ le nombre total de classes économiques (c'est-à-dire le nombre d'observations, en analyse de variance).

Ayant $J-1$ segments significativement différents, on teste la signification d'un $J$-ième segment en comparant la quantité :

$$
\Phi=(N-J)\left(\frac{\Delta J-1}{\Delta J}-1\right)
$$

à la valeur $F_{\alpha ; 1, N-J}$ prise par la fonction de Fisher-Snedecor à 1 et $N-J$ degrés de liberté, au seuil $\alpha$ (en général $\alpha$ est égal à 10\%,5\% ou $1 \%$ ).

Si $\Phi$ est plus grand, c'est-à-dire si :

$$
\frac{(N-J)(\Delta J-1 / \Delta J-1)}{F_{\alpha ; 1, N-J}}>1
$$

on conclut que le $J$-ième segment est significatif.

En analyse de variance, le $J$-ième segment est donné ; tandis qu'ici il est défini par l'échantillon. La loi de $\Phi$ n'est donc pas celle de FisherSnedecor. On peut néanmoins déduire, par analogie formelle, un critère pour le choix de $J$. Nous proposons de choisir $J$ parmi les valeurs qui donnent un maximum local au rapport

$$
\Phi / F_{\alpha ; 1, N-J}
$$


Au lieu de se rattacher au test du passage de $J-1$ à $J$, on peut aussi se rattacher à celui du passage de 1 à $J$; ce qui conduit à choisir $J$ parmi les maximums de :

$$
\frac{\frac{N-1}{J-1}(\Delta 1 / \Delta J-1)}{F_{\alpha ; J-1, N-J}}
$$
bons.

L'expérience semble montrer que ce critère donne des résultats moins

\subsection{2. - Espace à plusieurs dimensions}

Lorsque l'espace économique a une dimension $m>1$ et que les composantes d'un même vecteur sont indépendantes et de même variance, on peut raisonnablement définir la distance entre deux classes $\omega$ et $\omega^{\prime}$ à partir de la norme :

$$
\left\|y(\omega)-y\left(\omega^{\prime}\right)\right\|^{2}=\sum_{i=1}^{m}\left[y_{i}(\omega)-y_{i}\left(\omega^{\prime}\right)\right]^{2}
$$

En décomposant une distance en $m$ composantes aléatoirement indépendantes, on peut encore aboutir au critère :

$$
\frac{(N-J)(\Delta J-1 / \Delta J-1)}{F_{\alpha ; m, m(N-J)}}
$$

Si les composantes sont corrélées, mais qu'on connaît leur matrice des variances et co-variances $\Lambda$, en utilisant la norme :

$$
\|x\|^{2}=x^{\prime} \Lambda^{-1} x
$$

on aboutit encore au critère (13) ci-dessus.

S'il faut estimer la matrice $\Lambda$, il faudrait, en toute rigueur, faire appel aux résultats de l'analyse multivariée. Mais les formules obtenues ne sont pas simples. Dans la pratique, on pourra toujours se contenter du critère (13).

\section{2. - ASPEGT PRATIQUE}

Voici comment se rencontre le problème de segmentation en pratique.

\section{1. - Ensembles de variables}

On doit distinguer deux ensembles de variables : le premier ensemble est celui des variables descriptives; le second, celui des variables objectives.

2.1.1. - Variables descriptives. - Les variables descriptives servent à construire la population. S'il existe $S$ variables descriptives, chacune se eomposant de $N_{s}$ éventualités $(s=1, \ldots, S)$, alors le nombre des classes économiques est $N=N_{1} \times \ldots \times N_{S}$. L'ensemble des variables 
descriptives peut donc être considéré comme une seule variable $X$ présentant $N$ éventualités $X_{1}, \ldots, X_{N}$. Chaque éventualité $X_{n}(n=1, \ldots, N)$ est une classe économique.

2.1.2. - Variables objectives. - Les variables objectives permettent, par l'intermédiaire de la fonction objective, de définir l'homogénéité et la différenciation des divers segments en fonction de l'objectif fixé. S'il existe $Q$ variables objectives, chacune se composant de $R_{q}$ éventualités $(q=1, \ldots, Q)$, alors le nombre de dimensions de l'espace économique est :

$$
R=R_{1} \times \ldots \times R_{q} \times \ldots \times R_{Q} .
$$

L'ensemble des variables objectives peut donc être considéré comme une seule variable $Y$ présentant $R$ éventualités $Y^{1}, \ldots, Y^{R}$. Chaque éventualité $(r=1, \ldots, R)$ est une dimension de l'espace économique.

Remarque: Selon le problème, une même variable peut être descriptive ou objective. Dans l'exemple 3.1, l'âge est une variable descriptive, alors que dans l'exemple 3.3, l'âge est une variable objective.

\section{2. - Données du problème}

2.2.1. - Données brutes. - Les données brutes sont celles recueillies expérimentalement sur l'ensemble des variables descriptives et objectives. Elles se présentent en général sous la forme suivante:

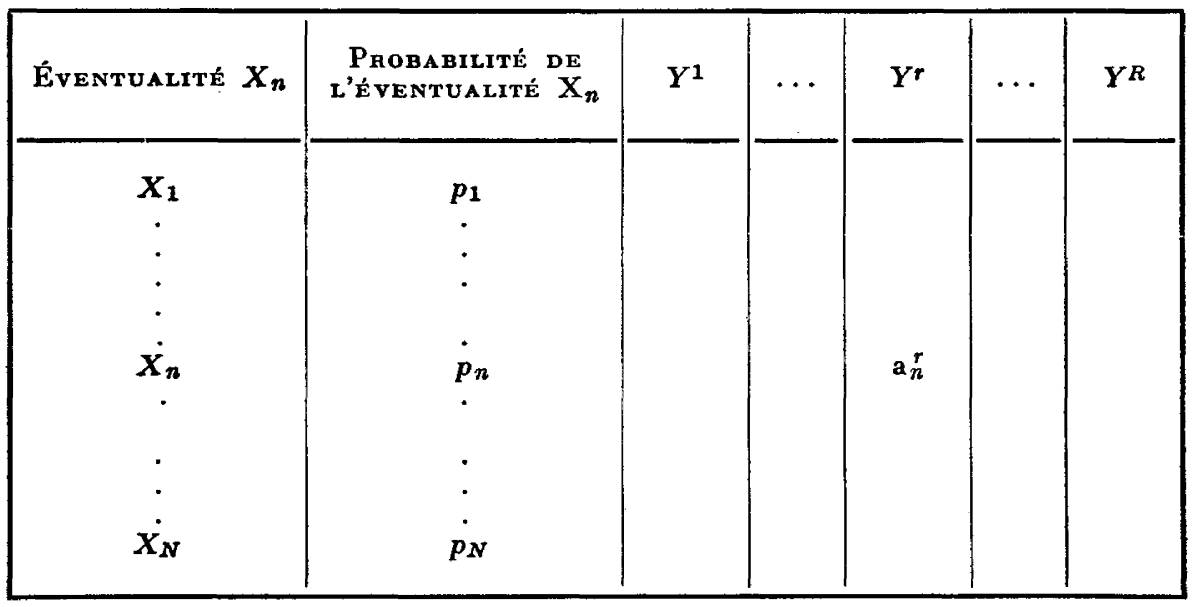

Dans ce tableau, $a_{n}^{r}$ est un nombre qui caractérise le couple $\left(X_{n}, Y r\right)$ (cf. les exemples au $\S 3$ ).

D'autre part, le plus souvent les éventualités $Y^{1}, \ldots, Y^{R}$ sont corrélées; dans les données brutes, peut donc se trouver une matrice de corrélation $\Lambda$.

2.2.2. - Données utiles. - De ces données brutes, il faut dégager les données utiles. La mesure (ou fréquence de répartition) de chaque éventualité $X_{n}$ peut être prise comme égale à sa probabilité : $p\left(X_{n}\right)=p_{n}$. 
Quant aux vecteurs économiques $y_{n}=\left(y_{n}^{r}\right)$, ils se déduisent des $a_{n}^{r}$ en les multipliant par des facteurs convenables (facteurs de pondération ou facteurs de normalisation). Enfin, la matrice de métrisation dépend de la corrélation entre les diverses éventualités $Y^{\mathbf{1}}, \ldots Y^{\boldsymbol{R}}$. Si ces éventualités sont orthogonales (c'est-à-dire non corrélées), on peut admettre que la matrice de métrisation est égale à l'unité. On peut alors démontrer que dans le cas général la matrice de métrisation est égale à l'inverse de la matrice de corrélation : $M=\Lambda^{-1}$.

\section{3. - EXEMPLES D'APPLICATIONS}

Nous allons donner quelques classes d'application.

\section{1. - Première classe}

Dans cette première classe, on est en présence d'une seule variable objective à une éventualité. Nous allons prendre un exemple typique : la segmentation du marché [1].

Il s'agit de segmenter un échantillon de 1000 Parisiennes sur l'utilisation d'un produit de beauté. Les critères de segmentation possibles sont l'âge (3 éventualités : $18-24 ; 25-34 ; 35-49)$ et la classe sociale ( 3 éventualités : pauvre, moyenne, riche). Le caractère choisi pour la segmentation est le niveau de consommation (quantité achetée par 100 Parisiennes).

3.1.1. - Variables. - Ici, les variables descriptives sont les critères de segmentation : âge, classe sociale. Comme chaque variable se compose de trois éventualités, le nombre de classes économiques est

$$
N=N_{1} \times N_{2}=3 \times 3=9 .
$$

Il n'y a qu'une seule variable objective : c'est le niveau de consommation ; cette variable ne présente qu'une seule éventualité $(R=1)$.

3.1.2. - Données. - Le Panel des consommateurs fournit le tableau suivant :

\begin{tabular}{|c|c|c|c|c|c|}
\hline$n$ & $\begin{array}{r}\text { Classe éco } \\
X_{n}\end{array}$ & VOMTQUE & $\begin{array}{c}\text { EFFECTif DE } \\
\text { LA Chasse : } \\
p_{n}\end{array}$ & $\begin{array}{c}\text { Quantités } \\
\text { ACHETÉES : } \\
a_{n}\end{array}$ & $\begin{array}{c}\text { NIVEAU DE } \\
\text { CONSOMMATION : } \\
Y_{n}\end{array}$ \\
\hline $\begin{array}{l}1 \\
2 \\
3 \\
4 \\
5 \\
6 \\
7 \\
8 \\
9\end{array}$ & $\begin{array}{l}\text { Riche, } \\
\text { Riche, } \\
\text { Riche, } \\
\text { Moyenne, } \\
\text { Moyenne, } \\
\text { Moyenne, } \\
\text { Pauvre, } \\
\text { Pauvre, } \\
\text { Pauvre, }\end{array}$ & $\begin{array}{l}18-24 \\
25-34 \\
35-49 \\
18-24 \\
25-34 \\
35-49 \\
18-24 \\
25-34 \\
35-49\end{array}$ & $\begin{array}{r}30 \\
34 \\
51 \\
36 \\
120 \\
209 \\
81 \\
216 \\
223\end{array}$ & $\begin{array}{l}14 \\
23 \\
25 \\
13 \\
38 \\
53 \\
20 \\
25 \\
27\end{array}$ & $\begin{array}{l}47 \\
68 \\
49 \\
36 \\
32 \\
26 \\
24 \\
11 \\
12\end{array}$ \\
\hline
\end{tabular}


Les premières colonnes sont des données brutes; la dernière colonne est calculée par $Y_{n}=\frac{a_{n}}{p_{n}} \times 100$. La fréquence de répartition est prise égale à l'effectif de la classe. La matrice de métrisation est égale à l'unité.

3.1.3. - Résultats. - L'algorithme de la segmentation agglomérative hiérarchique conduit au résultat suivant.

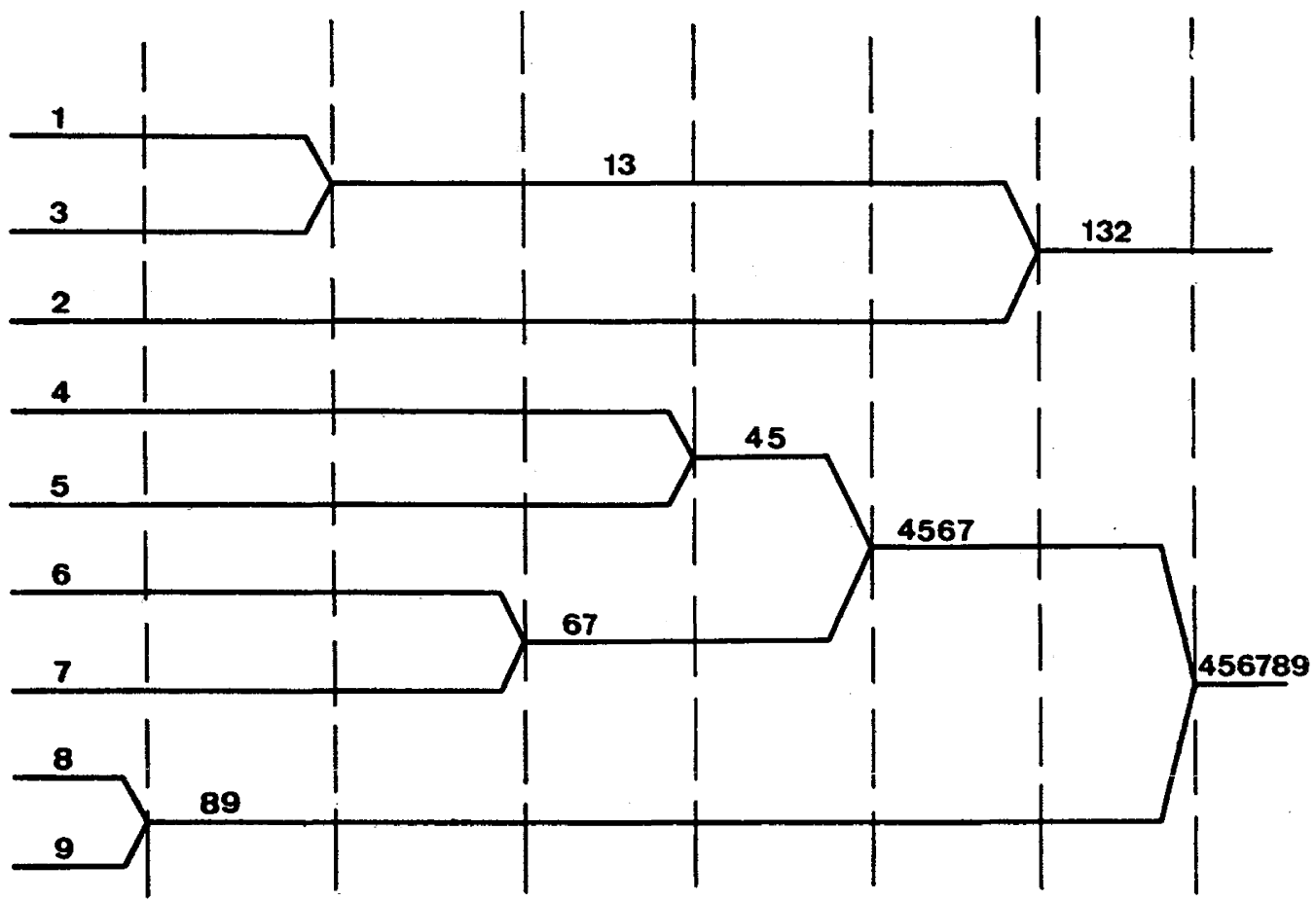

Figure 1

Le choix du nombre $J$ des segments se fait comme dans [18]. On trouve $J=5$. La 5 -segmentation obtenue est

$$
(1,3) ;(2) ;(4,5) ;(6,7) ;(8,9) \text {. }
$$

Les 5 segments sont donc :

$$
\begin{aligned}
& \text { I : riche, } 25-34 \text { ans } \\
& \text { II : riche, } 18-24 \text { ans, } 35-49 \text { ans } \\
& \text { III : moyenne, } 18-24 \text { ans } \\
& \text { IV : moyenne, } \\
& 35-49 \text { ans ; pauvre, 18-24 ans } \\
& \text { V : pauvre, } 25-49 \text { ans. }
\end{aligned}
$$




\section{2. - Deuxième classe}

Dans cette classe d'applications, il y a plusieurs variables objectives dont chacune ne présente qu'une éventualité. Comme exemples : la segmentation de l'ensemble des employés d'une usine suivant leurs qualités, de l'ensemble des produits pétroliers suivant leurs propriétés, du marché suivant plusieurs caractères. L'exemple typique est l'étude. anthropométrique des tribus indiennes [12].

On désire faire une segmentation de l'ensemble de douze tribus indiennes selon les neufs caractères suivants :

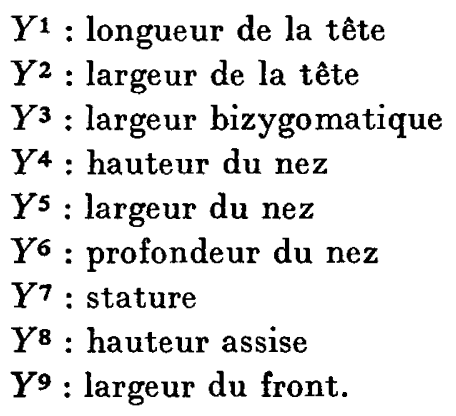

3.2.1. - Variables. - Ici, il y a une seule variable descriptive : le nom des tribus indiennes, se composant d'éventualités : Basti, Brahmin... Par contre, il existe 9 variables objectives, chacune présentant une seule éventualité. La dimension de l'espace objectif est donc $R=9 \times 1=9$.

3.2.2. - Données brutes. - Les données brutes sont rassemblées dans le tableau suivant:

\begin{tabular}{|c|c|c|c|c|c|c|c|c|c|c|c|}
\hline$n$ & Tribus & $\begin{array}{c}\text { EFFEC- } \\
\text { TIFS }\end{array}$ & $Y^{1}$ & $Y^{2}$ & $Y^{3}$ & $Y^{4}$ & $Y^{5}$ & $Y^{6}$ & $Y^{7}$ & $Y^{8}$ & $Y^{9}$ \\
\hline $\begin{array}{r}1 \\
2 \\
3 \\
4 \\
5 \\
6 \\
7 \\
8 \\
9 \\
10 \\
11 \\
12\end{array}$ & $\begin{array}{l}\text { Basti .... } \\
\text { Brahmin } \\
\text { Chattri. . } \\
\text { Muslim... } \\
\text { Bhatu.... } \\
\text { Habru ... } \\
\text { Bril. ..... } \\
\text { Dom. .... } \\
\text { Ahir ..... } \\
\text { Kurmi ... } \\
\text { Kahar . . } \\
\text { Autres } \\
\text { tribus. . }\end{array}$ & $\begin{array}{r}86 \\
92 \\
139 \\
168 \\
150 \\
124 \\
186 \\
113 \\
68 \\
94 \\
57 \\
\\
173\end{array}$ & $\begin{array}{l}191,92 \\
191,35 \\
192,58 \\
190,78 \\
186,10 \\
181,94 \\
181,87 \\
186,87 \\
187,45 \\
188,86 \\
188,83 \\
187,69\end{array}$ & $\begin{array}{l}139,88 \\
139,50 \\
131,72 \\
137,40 \\
138,58 \\
137,40 \\
137,62 \\
137,52 \\
138,12 \\
137,86 \\
136,28 \\
\\
136,84\end{array}$ & $\begin{array}{l}133,36 \\
132,68 \\
131,70 \\
131,40 \\
133,58 \\
131,16 \\
131,18 \\
132,64 \\
131,70 \\
131,82 \\
130,70 \\
131,30\end{array}$ & $\begin{array}{l}51,24 \\
50,40 \\
52,72 \\
51,38 \\
52,06 \\
50,30 \\
48,60 \\
50,34 \\
48,98 \\
49,22 \\
48,62 \\
\\
48,72\end{array}$ & $\begin{array}{l}36,55 \\
36,13 \\
35,64 \\
36,36 \\
35,65 \\
35,82 \\
37,49 \\
38,11 \\
35,60 \\
36,21 \\
36,51 \\
\\
36,27\end{array}$ & $\begin{array}{l}25,49 \\
24,74 \\
24,73 \\
24,49 \\
25,09 \\
24,19 \\
24,05 \\
25,33 \\
24,19 \\
24,03 \\
24,08 \\
23,13\end{array}$ & $\begin{array}{l}164,51 \\
165,07 \\
163,33 \\
162,45 \\
163,38 \\
164,91 \\
162,92 \\
166,53 \\
161,37 \\
161,35 \\
160,53 \\
161,34\end{array}$ & $\begin{array}{l}86,43 \\
86,25 \\
82,25 \\
81,83 \\
84,49 \\
85,53 \\
82,60 \\
84,19 \\
84,35 \\
83,41 \\
81,47 \\
83,09\end{array}$ & $\begin{array}{r}104,74 \\
104,46 \\
103,98 \\
103,28 \\
99,34 \\
100,18 \\
103,36 \\
104,16 \\
12,76 \\
102,62 \\
101,68 \\
102,44\end{array}$ \\
\hline
\end{tabular}


Ces neufs caractères sont corrélés; la matrice de corrélation est :

\begin{tabular}{|c|c|c|c|c|c|c|c|c|c|}
\hline & $Y^{1}$ & $Y^{2}$ & $Y^{3}$ & $Y^{4}$ & $Y^{5}$ & $Y^{6}$ & $Y^{7}$ & $Y^{8}$ & $Y^{9}$ \\
\hline$Y^{1}$ & 1 & 0,20 & 0,28 & 0,18 & 0,19 & 0,15 & 0,27 & 0,27 & 0,23 \\
\hline$Y^{2}$ & & 1 & 0,54 & 0,17 & 0,14 & 0,13 & 0,19 & 0,21 & 0,45 \\
\hline$Y^{3}$ & & & 1 & 0,19 & 0,27 & 0,16 & 0,29 & 0,30 & 0,49 \\
\hline$Y^{4}$ & & & & 1 & 0,04 & 0,29 & 0,20 & 0,22 & 0,11 \\
\hline$Y^{5}$ & & & & & 1 & 0,11 & 0,14 & 0,12 & 0,18 \\
\hline$Y^{6}$ & & & & & & 1 & 0,18 & 0,21 & 0,12 \\
\hline$Y^{7}$ & & & & & & & 1 & 0,58 & 0,22 \\
\hline$Y^{8}$ & & & & & & & & 1 & 0,30 \\
\hline$Y^{9}$ & & & & & & & & & 1 \\
\hline
\end{tabular}

3.2.3. - Données utiles. - La fréquence de répartition de chaque classe économique est égale à leur effectif. Pour obtenir les vecteurs économiques, nous utilisons les variables centrées réduites :

$$
Y_{n}^{r}=\frac{Y_{n}^{r}-m\left(Y^{r}\right)}{\sigma\left(Y^{r}\right)}
$$

où $m\left(Y^{r}\right)$ et $\sigma\left(Y^{r}\right)$ sont respectivement la moyenne et l'écart-type de $Y^{r}$. La matrice de métrisation est prise égale à l'inverse de la matrice de corrélation.

3.2.4. - Résultats. - La segmentation se fait alors aisément appliquant la méthode indiquée dans [18], on trouve que :

(i) le nombre de segments qu'il faut prendre est $J=5$.

(ii) la 5-segmentation obtenue est Basti-Brahmin; Chattri-Muslim; Bhatu-Habru ; Bril-Dom ; Ahir-Kurmi - Kahar - autres tribus.

Nota : Pratiquement, on procède autrement. On remplace les caractères donnés par des caractères fictifs et orthogonaux (et réduits), et on prend la matrice de métrisation égale à l'unité. Ce procédé a été programmé sur ordinateur. 


\section{3. - Troisième classe d'application}

Dans cette classe, il existe plusieurs variables objectives dont chacune présente diverses éventualités. L'exemple typique est la typologie de périodiques basée sur l'analyse de leur clientèle.

3.3.1. - Variables. - Il n'existe qu'une seule variable descriptive : c'est le titre (50 éventualités : Réalité, la Maison Française, Historia,... Clair Foyer). Les variables objectives sont nombreuses et chacune d'elles présente plusieurs éventualités. Pour simplifier, nous supposons qu'il n'existe que deux variables objectives.

Sexe : deux éventualités (masculin, féminin).

Age : trois éventualités $(15-30 ; 31-50 ; 51-80)$.

Alors, la dimension de l'espace économique est $R=2 \times 3=6$.

Autrement dit, chaque vecteur économique possède six composantes :

$Y^{1}$ : hommes de 15 à 30 ans ; $\quad Y^{4}$ : femmes de 15 à 30 ans ;

$Y^{2}$ : hommes de 31 à 50 ans; $\quad Y^{5}:$ femmes de 31 à 50 ans ;

$Y^{3}$ : hommes de 51 à 80 ans ; $\quad Y^{6}$ : femmes de 51 à 80 ans.

3.2.2. - Données brutes. - Les données brutes sont contenues dans le tableau suivant :

\begin{tabular}{|c|c|c|c|c|c|c|c|c|}
\hline $\mathrm{n}$ & Trtre & $\begin{array}{l}\text { Total Des } \\
\text { Lecteurs }\end{array}$ & $Y^{1}$ & $Y^{2}$ & $Y^{3}$ & $Y^{4}$ & $Y^{5}$ & $Y^{6}$ \\
\hline $\begin{array}{l}1 \\
2 \\
3\end{array}$ & $\begin{array}{l}\text { Réalité } \ldots \ldots \ldots \ldots \\
\text { Maison française } \ldots \\
\text { Historia } \ldots \ldots \ldots \ldots\end{array}$ & $\begin{array}{ll}1 & 500 \\
2 & 000 \\
2 & 500\end{array}$ & $\begin{array}{l}100 \\
200 \\
300\end{array}$ & $\begin{array}{l}200 \\
100 \\
400\end{array}$ & $\begin{array}{l}500 \\
300 \\
900\end{array}$ & $\begin{array}{l}200 \\
500 \\
100\end{array}$ & $\begin{array}{l}100 \\
500 \\
300\end{array}$ & $\begin{array}{l}400 \\
400 \\
500\end{array}$ \\
\hline $\begin{array}{l}49 \\
50\end{array}$ & $\begin{array}{l}\text { Télé } 7 \text { jours..... } \\
\text { Télémagazine ... }\end{array}$ & $\begin{array}{l}2000 \\
1000\end{array}$ & $\begin{array}{r}100 \\
50\end{array}$ & $\begin{array}{l}200 \\
100\end{array}$ & $\begin{array}{l}200 \\
100\end{array}$ & $\begin{array}{l}400 \\
200\end{array}$ & $\begin{array}{l}500 \\
250\end{array}$ & $\begin{array}{l}600 \\
300\end{array}$ \\
\hline
\end{tabular}

3.2.3. - Données utiles. - Chaque titre est une classe économique dont la fréquence de répartition $p_{n}$ est égale au nombre de lecteurs de ce titre. Pour obtenir les vecteurs économiques, on divise chaque colonne par la première colonne :

$$
Y_{n}^{r}=\frac{Y_{n}^{r}}{p_{n}}
$$

En effet, on fait la segmentation non pas sur le nombre de lecteurs, mais sur la proportion des lecteurs (dans l'exemple donné, Télé 7 jours et Télémagazine doivent être considérés, comme ayant une dispersion de mélange nulle). Pour plus de détail, cf. [18]. 
Nota: Bergonier et Boucharenc [2] ont donné une autre méthode basée sur la théorie de l'information, les deux méthodes donnent sensiblement les mêmes résultats.

\section{SOURCES REFERENTIELLES}

[1] Agostini (J. M.), Une méthode de segmentation du marché, Esomar-Wapor Conférence (1966), Dublin,

[2] Bergonier (H.) et Boucharenc (L.), Une méthode de segmentation basée sur la théorie de l'information, Prix Marcel Dassault (1966).

[3] Cox (D. R.), Note on grouping J. Amer. Stat. Ass. 52 (1957), 543-547.

[4] Darenrus (T.), The problem of optimum stratification, Skandinavisk Aktnarietidskrift (1950), 203-213.

[5] Dalenius (T.) et Hogdes (J. L. Fr.), Minimum variance stratification J. Amer. Strat. Assoe. 54 (1959), 88-101.

[6] Fischer (W.), On grouping for maximum homogeneity, J. Amer. Stat. Assoc. 53 (1958), 789-798.

[7] Green (P. E.), Frank (R. E.) et Robinson (P. J.), Cluster Analysis in Test Market Selection Management Science. B, 13 (1967), 387-400.

[8] GuInet (J.), Recherche d'une segmentation optimale, AUROC (1966).

[9] Howard (R. N.), Classifying a population into homogen groups ESOMARWAPOR Conference (1965), Cambridge.

[10] Jizba, A Contribution to statistical theory of classification, Standford Univ. Publ. Geol. Sci. 9 (1964), 729-756.

[11] MacNaughton-Smith (P.), Williams (W. T.) et Dale (M. B.), On objective method of weighting in similarity analysis, Nature, 201 (1964), 426.

[12] NGHiEM (Ph. T.), La segmentation aux moindres carrés et l'analyse de la variance D.E., CEGOS (1968).

[13] RAo (C.R.), Advanced statistical methods in biometric research, John Wiley and Sons, New York (1952).

[14] Sneath (Ph. A) et Sokal (R.R.), Principles of numerical taxonomy, Freeman, San Francisco et Londres (1963).

[15] Thorndike (R.L.), Who belongs to the family? Psychometrika, 18 (1953), 267-276.

[16] Vo-Krac (K), Segmentation aux moindres carrés dans un espace pré-hilbertien, C. R. Acad. Sci. Paris, novembre 1967.

[17] Vo-Krac (K), Étude théorique du problème de segmentation, D. E. CEGOS, 1966.

[18] Vo-Kнас (K), Applications pratiques de la segmentation par la méthode des moindres carrés, D. E. CEGOS, 1966-1967.

[19] Yaglom (A. M.) et Yaglom (I. M.), Probabilité et information, Dunod, Paris (1959).

[20] Document de synthèse sur la méthode de segmentation aux moindres carrés D. E. CEGOS (1967). 